\title{
Diagnostic Utility of Optic Nerve Measurements with MRI in Patients with Optic Nerve Atrophy
}

\author{
(D) B. Zhao, (D) N. Torun, (D) M. Elsayed, (D)A.-D. Cheng, (D) A. Brook, (D).-M. Chang, and (D) R.A. Bhadelia
}

\begin{abstract}
BACKGROUND AND PURPOSE: No MR imaging measurement criteria are available for the diagnosis of optic nerve atrophy. We determined a threshold optic nerve area on MR imaging that predicts a clinical diagnosis of optic nerve atrophy and assessed the relationship between optic nerve area and retinal nerve fiber layer thickness measured by optical coherence tomography, an ancillary test used to evaluate optic nerve disorders.
\end{abstract}

MATERIALS AND METHODS: We evaluated 26 patients with suspected optic nerve atrophy ( 8 with unilateral, 13 with bilateral and 5 with suspected but not demonstrable optic nerve atrophy) who had both orbital MR imaging and optical coherence tomography examinations. Forty-five patients without optic nerve atrophy served as controls. Coronal inversion recovery images were used to measure optic nerve area on MR imaging. Retinal nerve fiber layer thickness was determined by optical coherence tomography. Individual eyes were treated separately; however, bootstrapping was used to account for clustering when appropriate. Correlation coefficients were used to evaluate relationships; receiver operating characteristic curves, to investigate predictive accuracy.

RESULTS: There was a significant difference in optic nerve area between patients' affected eyes with optic nerve atrophy (mean, $3.09 \pm$ $1.09 \mathrm{~mm}^{2}$ ), patients' unaffected eyes (mean, $5.27 \pm 1.39 \mathrm{~mm}^{2} ; P=.008$ ), and control eyes (mean, $6.27 \pm 2.64 \mathrm{~mm}^{2} ; P<.001$ ). Optic nerve area $\leq 4.0 \mathrm{~mm}^{2}$ had a sensitivity of 0.85 and a specificity of 0.83 in predicting the diagnosis of optic nerve atrophy. A significant relationship was found between optic nerve area and retinal nerve fiber layer thickness $(r=0.68, P<.001)$.

CONCLUSIONS: MR imaging-measured optic nerve area $\leq 4.0 \mathrm{~mm}^{2}$ has moderately high sensitivity and specificity for predicting optic nerve atrophy, making it a potential diagnostic tool for radiologists.

ABBREVIATIONS: OCT = optical coherence tomography; $\mathrm{ONA}=$ optic nerve atrophy; $\mathrm{ON}_{\text {area }}=$ optic nerve area; $\mathrm{RNFL}=$ retinal nerve fiber layer; $\mathrm{ROC}=$ receiver operating characteristic

O ptic nerve atrophy (ONA) occurs when there is injury to the retinal ganglion cells or their axons and, at present, is primarily diagnosed on a clinical basis. ${ }^{1,2}$ Careful assessment, taking into account the history and demographic profile of the patient and the clinical examination (including visual acuity, color vision, pupils, and the fundus), combined with specific ancillary testing such as computerized visual fields, is necessary to establish the diagnosis and etiology. However, it has been previously shown that the clinical diagnosis of ONA may not be reliable in the hands of clinical practitioners without specialized training in neuro-

Received September 4, 2018; accepted after revision January 5, 2019.

From the Departments of Radiology (B.Z., M.E., A.B., Y.-M.C, R.A.B.) and Ophthalmology (N.T., A.-D.C.), Beth Israel Deaconess Medical Center, Boston, Massachusetts.

Please address correspondence to Brian Zhao, MD, Department of Radiology, Beth Israel Deaconess Medical Center, WCB90, 330 Brookline Ave, Boston, MA, 02215;

e-mail: bzhao@bidmc.harvard.edu

http://dx.doi.org/10.3174/ajnr.A5975 ophthalmology. ${ }^{3}$ Because specialists with such training are usually limited to tertiary care centers, a final diagnosis may require additional referrals to these centers.

The classic clinical presentation of ONA includes decreased visual acuity, visual fields, and color vision. ${ }^{1,4}$ It is our experience that patients with such vision-related symptoms are often referred for orbital MR imaging examinations before they are seen by a neuro-ophthalmologist. This procedure puts radiologists in a unique frontline position of assessing the optic nerves before a final diagnosis of ONA is made. While the diagnosis of ONA can usually be suggested on MR imaging by observing the decreased size of the intraorbital optic nerve, there are no definite measurement criteria currently available to the radiologist. We believe that for MR imaging-based optic nerve measurements to be clinically useful, such measurements need to be validated, with the final diagnosis of ONA made by a neuro-ophthalmologist based on clinical examination and retinal nerve fiber layer (RNFL) thickness measured on optical coherence tomography (OCT), which 
correlates with axonal loss and is routinely used in ophthalmologic practice in the initial and follow-up assessment of various optic nerve disorders.

Based on this background information, our purpose was the following: 1) to determine a threshold optic nerve area $\left(\mathrm{ON}_{\text {area }}\right)$ on MR imaging that predicts the clinical diagnosis of ONA in the adult population made by a neuro-ophthalmologist, and 2) to assess the relationship between $\mathrm{MR}$ imaging-measured $\mathrm{ON}_{\text {area }}$ and RNFL thickness measured by OCT.

\section{MATERIALS AND METHODS \\ Patients}

Institutional review board approval was obtained for this Health Insurance Portability and Accountability Act-compliant retrospective study with a waiver of informed consent. Patients diagnosed with optic nerve atrophy by a neuro-ophthalmologist at a single institution between June 2009 and December 2016 were identified. Twenty-six adult patients who had both clinical and OCT examinations and an orbital MR imaging within 1 month of each other were included. The diagnostic criteria used for optic nerve atrophy were the following: 1) clinical examination unequivocally consistent with optic nerve atrophy, and 2) RNFL thickness $<85 \mu \mathrm{m}$. Among these 52 patient eyes, a total of 34 "affected" eyes with an unequivocal clinical diagnosis of ONA and 18 "unaffected" eyes were used for analysis. The patient population consisted of 14 women and 12 men. The mean age was $58.2 \pm$ 14.9 years (range, $32-89$ years).

\section{Controls}

The controls were collected in the following manner: 1) 18 unaffected eyes of the patients, and 2) 90 "healthy" eyes from 35 consecutive patients undergoing imaging for seizures, which included coronal inversion recovery images that provided details of optic nerves, and 10 patients evaluated for suspected orbital abnormalities but had no findings suggestive of ONA on neuro-ophthalmologic examination. The healthy eye population consisted of 20 women and 25 men. The mean age was $55.2 \pm 11.9$ years (range, 27-77 years).

\section{MR Imaging}

MR imaging examinations were performed on 1 of two 1.5T Signa HDx MR imaging scanners (GE Healthcare Milwaukee, Wiscon$\sin$ ). The MR imaging parameters for patients and controls with visual symptoms not related to ONA were the following: TR = $6600 \mathrm{~ms}, \mathrm{TE}=85 \mathrm{~ms}, \mathrm{TI}=50 \mathrm{~ms}$, slice thickness $=3 \mathrm{~mm}, \mathrm{FOV}=$ 18 , matrix $=320 \times 320$. The MR imaging parameters for controls belonging in the seizure cohort were the following: $\mathrm{TR}=6000 \mathrm{~ms}$, $\mathrm{TE}=80 \mathrm{~ms}$, TI $=50 \mathrm{~ms}$, slice thickness $=2.5 \mathrm{~mm}, \mathrm{FOV}=22$, matrix $=320 \times 320$.

For both patients and controls, images from a coronal inversion recovery sequence were used to measure $\mathrm{ON}_{\text {area }}$ (excluding the optic nerve sheath) at an image slice along the intraorbital portion of the optic nerve. The image was chosen on the basis of where the optic nerves appeared most round and most perpendicular to the coronal plane based on visual inspection, approximately halfway between the optic nerve-globe junction and the orbital apex. Measurements were obtained from the same image

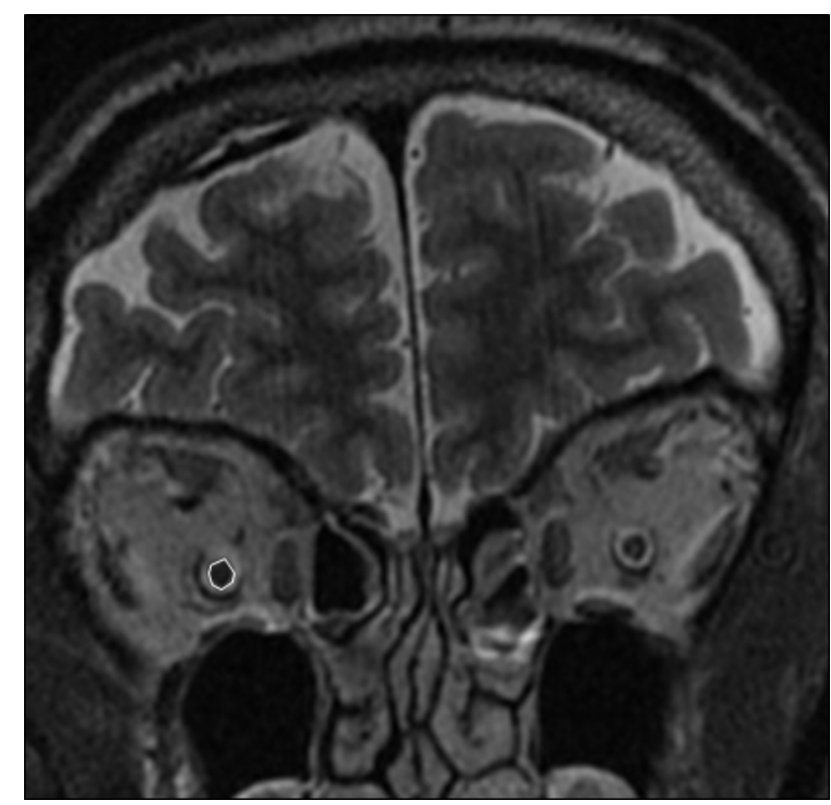

FIG 1. MR imaging measurements of the optic nerve area. Coronal inversion recovery image through the orbits approximately midway between the orbital apex and globes. The measurement of the crosssectional area of the right optic nerve (excluding the optic nerve sheath) is shown using a freeform ROI measurement tool on the PACS. The left optic nerve is not outlined for comparison.

slice by 2 of the coauthors (radiology residents who received training in $\mathrm{ON}_{\text {area }}$ measurements from the senior author with $>25$ years' experience) independently and blinded to whether the study belonged to a patient or a control.

A freeform ROI measurement tool on the PACS was used to outline and measure the cross-sectional optic nerve areas (Fig 1). The optic nerve area measurement process involved zooming in on each nerve separately to facilitate precise and accurate outlining of the nerve contours.

\section{OCT}

RNFL thickness was determined on a Spectralis SD OCT machine (Heidelberg Engineering, Heidelberg, Germany), and an average RNFL thickness was calculated for each eye. RNFL thickness measurements were available for the entire patient population of 26 individuals for both diseased and nondiseased eyes. The control population did not have any RNFL thickness measurements because they did not have clinical concerns to warrant OCT.

\section{Statistical Analyses}

Individual eyes were treated separately; however, bootstrapping was used to account for clustering when appropriate. The average of $\mathrm{ON}_{\text {area }}$ measurements between the 2 readers was used for all analyses and comparisons, except for the discussion of interreader variability. Correlation coefficients were used to evaluate relationships; ANOVA with a Tukey post hoc procedure or $t$ test, to compare measurements; a $\chi^{2}$ test, to compare sex distribution; and a receiver operating characteristic (ROC) curve, to investigate predictive accuracy. Calculations were performed using Matlab 9.3 (MathWorks, Natick, Massachusetts). $P$ values $\leq .05$ were used to determine statistical significance.

AJNR Am J Neuroradiol 40:558-61 Mar 2019 www.ajnr.org 
Comparison of MRI-measured optic nerve area between patients' affected and unaffected eyes and healthy eyes of controls and comparison of RNFL thickness between patients' affected and unaffected eyes $^{\mathrm{a}}$

\begin{tabular}{lccc}
\hline & \multicolumn{3}{c}{ Optic Nerves } \\
\cline { 2 - 4 } & $\begin{array}{c}\text { Patients' } \\
\text { Affected } \\
(\boldsymbol{n}=\mathbf{3 4})\end{array}$ & $\begin{array}{c}\text { Patients' } \\
\text { Unaffected } \\
(\boldsymbol{n}=\mathbf{1 8})\end{array}$ & $\begin{array}{c}\text { Healthy } \\
(\boldsymbol{n}=\mathbf{9 0})\end{array}$ \\
\hline RNFL $(\mu \mathrm{m})$ & $67.12 \pm 13.55$ & $94.00 \pm 8.66$ & $\mathrm{NA}$ \\
& & $P_{\mathrm{p}}<.001$ & \\
Optic nerve area $\left(\mathrm{mm}^{2}\right)$ & $3.09 \pm 1.09$ & $5.27 \pm 1.39$ & $6.27 \pm 2.64$ \\
& & $P_{\mathrm{p}}=.008$ & $P_{\mathrm{c}}<.001$ \\
& & & $P_{\mathrm{uc}}=.21$ \\
\hline
\end{tabular}

Note:-NA indicates not applicable; $P_{c}$, comparison between patients' affected optic nerves and control optic nerves; $P_{\mathrm{p}}$, comparison between patients' affected and unaffected optic nerves; $P_{\text {uc }}$, comparison between patients' unaffected optic nerves and control optic nerves.

${ }^{a}$ Data are mean \pm SD unless otherwise indicated. No OCT data were available for the 90 healthy eyes in the control population because these individuals did not have clinical symptoms to warrant OCT measurement.

\section{RESULTS}

There was no statistically significant difference in $\operatorname{sex}(P=.44)$ or age $(P=.35)$ between the patient and control groups. No statistically significant relationship was found between $\mathrm{ON}_{\text {area }}$ and age in the adult population of this study $(r=-0.1, P=.23)$. There was a high correlation $(r=0.87)$ between the 2 readers in measuring the $\mathrm{ON}_{\text {area }}$. The mean difference in $\mathrm{ON}_{\text {area }}$ between the 2 observers was $0.93 \mathrm{~mm}^{2}$ with a within-subject SD of $1.2 \mathrm{~mm}^{2}$.

The Table summarizes the $\mathrm{ON}_{\text {area }}$ and RNFL thickness in patients' affected and unaffected eyes and $\mathrm{ON}_{\text {area }}$ measurements in healthy eyes. There was a statistically significant difference in RNFL thickness between the patients' affected and unaffected eyes $(P<.001)$.

There was a statistically significant difference in $\mathrm{ON}_{\text {area }}$ among patients' eyes with ONA (mean, $3.09 \pm 1.09 \mathrm{~mm}^{2}$ ), patients' unaffected eyes (mean, $5.27 \pm 1.39 \mathrm{~mm}^{2} ; P=.008$ ), and control eyes (mean, $6.27 \pm 2.64 \mathrm{~mm}^{2} ; P<.001$ ). No significant difference in $\mathrm{ON}_{\text {area }}$ was observed between patients' unaffected eyes and control eyes $(P=.21)$.

An ROC curve was created to test the ability of $\mathrm{ON}_{\text {area }}$ to predict the diagnosis of optic nerve atrophy, namely its ability to separate 34 affected eyes from 90 healthy eyes (Fig 2). The area under the curve was 0.91 . Selecting a threshold MR imagingmeasured $\mathrm{ON}_{\text {area }} \leq 4.0 \mathrm{~mm}^{2}$ yielded a sensitivity of 0.85 and a specificity of 0.83 in predicting the presence of ONA by clinical diagnosis.

A statistically significant positive relationship was found (Fig 3) between MR imaging-measured $\mathrm{ON}_{\text {area }}$ and RNFL thickness measured by OCT $(r=0.68, P<.001)$.

\section{DISCUSSION}

In our investigation of optic nerve area measured by MR imaging, a considerable variability between patients and controls and between eyes in the same individual was observed, as reported previously. $^{2}$ Nonetheless, a statistically significant difference in $\mathrm{ON}_{\text {area }}$ was observed between patients' diseased eyes, patients' unaffected eyes, and healthy eyes. Our analysis also showed that measurement of $\mathrm{ON}_{\text {area }}$ by $\mathrm{MR}$ imaging can be used clinically with very good diagnostic accuracy. ${ }^{5}$ We also observed a statistically significant relationship between MR imaging-measured

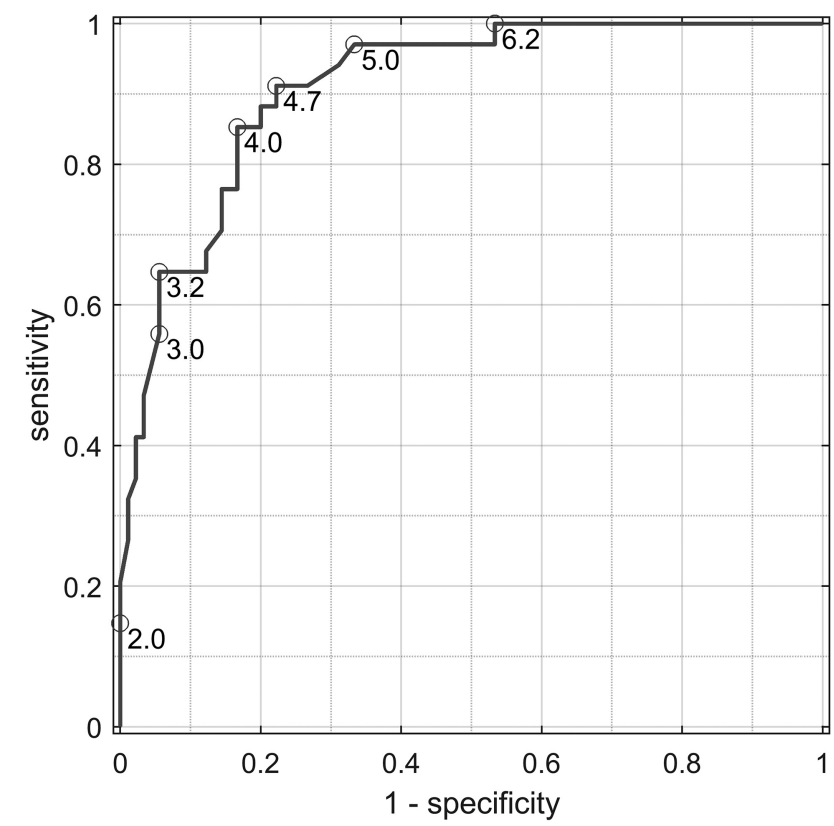

FIG 2. ROC curve showing the ability of $O \mathrm{~N}_{\text {area }}$ to predict the diagnosis of optic nerve atrophy. The ROC curve shows the ability to distinguish 34 affected eyes from 90 healthy eyes on the basis of the $\mathrm{ON}_{\text {area }}$. Using a threshold MR imaging-measured $\mathrm{ON}_{\text {area }} \leq 4.0 \mathrm{~mm}^{2}$ yields a sensitivity of 0.85 , specificity of 0.83 , and an AUC of 0.91 .

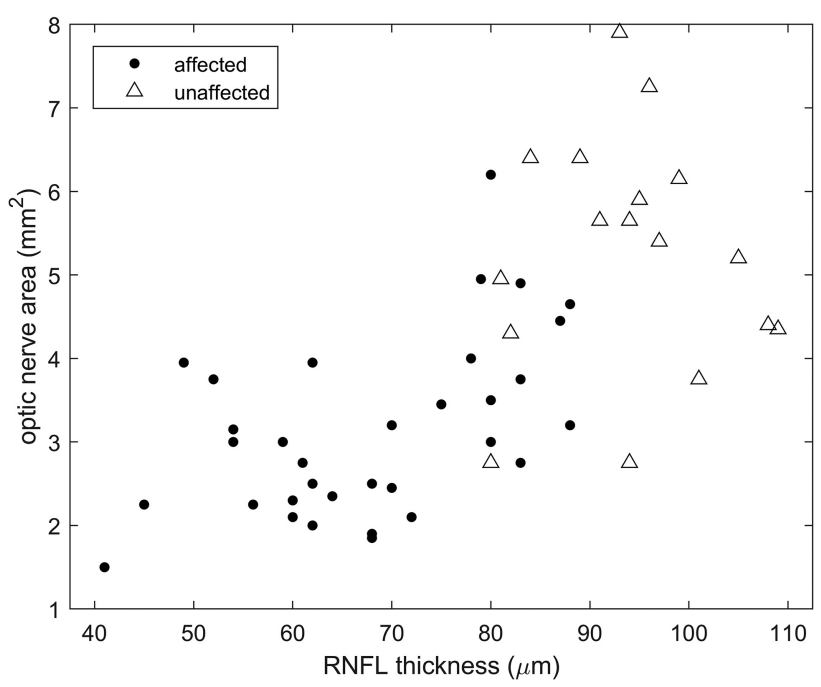

FIG 3. The relationship between MR imaging-measured optic nerve area and OCT-measured RNFL thickness. The scatterplot demonstrates a positive correlation between MR imaging-measured optic nerve area and OCT-measured RNFL thickness in patients with optic nerve atrophy. Circle data points denote those eyes with a clinical diagnosis of optic nerve atrophy. Triangle data points denote the unaffected eyes in the patient population (correlation coefficient $r=$ 0.68; 95\% Cl, 0.50-0.80; $P<.001$ ).

$\mathrm{ON}_{\text {area }}$ and RNFL thickness measured by OCT. This is consistent with the understanding of the pathophysiology of optic nerve atrophy - that is, the damage to retinal ganglion cells can cause anterograde (Wallerian) degeneration of the optic nerve or direct damage to the optic nerve can cause retrograde degeneration of the retinal ganglion cells. . $^{2,6,6}$

The optic nerve comprises the coalesced, myelinated axons of retinal ganglion cells. These axons are unmyelinated as they tra- 
verse within the retina toward the optic disc, comprising the retinal nerve fiber layer. ${ }^{8}$ Optic nerve atrophy occurs when there is injury to these ganglion cells or their axons. This process can result from various underlying etiologies, which include optic neuritis commonly associated with multiple sclerosis and various other optic neuropathies, such as ischemic (hypertension, diabetes, giant-cell arteritis); compressive (orbital or intracranial mass); and inflammatory, toxic, traumatic, or hereditary (mitochondrial disease) causes. ${ }^{1,4}$ Irrespective of the mechanism, loss of retinal ganglion cells or their axons results in irreversible atrophy, which manifests clinically in the form of vision impairment. ${ }^{2}$ The degree of optic atrophy depends entirely on the extent of damage to the optic nerve and is independent of the underlying etiology.

As previously mentioned, optic nerve atrophy remains primarily a clinical diagnosis. Specific vision testing and RNFL measurements via OCT have been used as adjunctive tools to establish the diagnosis and monitor disease progression. ${ }^{4,7,9-11}$ The results of this study suggest that optic nerve area measurements made on orbital MR imaging can also play a role in detecting and potentially diagnosing optic nerve atrophy and provide radiologists a role in identifying these patients and referring their care to an appropriate specialist.

The measurement of optic nerve area is a manual process, which raises concerns for interobserver variability. One element of variability depends on where the optic nerve is measured along its intraorbital segment. It has previously been demonstrated that optic nerve size significantly decreases from the globe to the orbital apex. ${ }^{12}$ To minimize this feature, we measured the optic nerve area at the same intraorbital location (approximately halfway between the optic nerve-globe junction and the orbital apex) in both patients and controls. The difference between the measurements of the 2 independent observers was noteworthy with a mean difference of $0.93 \mathrm{~mm}^{2}$ and a within-subject SD of $1.2 \mathrm{~mm}^{2}$. However, it can be reasonably expected that interobserver variability can be minimized by adhering to basic guidelines for the measurement technique. Furthermore, we also believe that increasing the resolution of images from $1 \mathrm{~mm}^{2}$ used in this study can also improve detection of optic nerve margins.

Optic nerve area (excluding the optic nerve sheath) was chosen as the main metric of measurement, as opposed to optic nerve diameter. During the measurement process, it was observed that many optic nerves and in particular those carrying a clinical diagnosis of optic nerve atrophy were not circular when viewed in cross-section. Many were ellipsoid, thus allowing varying diameter measurements. Thus, it was thought that measuring the total cross-sectional area would more accurately represent actual optic nerve size.

Optical coherence tomography is an established tool in ophthalmology and neurology and is used as an ancillary test to aid in the diagnosis of many ophthalmic diseases, including ONA. ${ }^{10}$
OCT uses measurement of the TE delay of backscattered infrared light via an interferometer and a low-coherence light source and quantifies RNFL thickness. ${ }^{2,9}$ The association between measurable thinning of the RNFL in patients with optic nerve atrophy has been previously observed. ${ }^{11}$

The moderately strong positive correlation found between MR imaging-measured $\mathrm{ON}_{\text {area }}$ and OCT-measured RNFL thickness is consistent with intuitive expectations, because they represent different regions of the same underlying cellular structures. ${ }^{8}$ This finding also corroborates the conclusions from prior investigations into this relationship ${ }^{2}$ and further establishes orbital MR imaging as a method of assessing for optic nerve pathology relating to atrophy.

\section{CONCLUSIONS}

Our data suggest that an MR imaging-measured optic nerve area of $\leq 4.0 \mathrm{~mm}^{2}$ has both strong sensitivity and high specificity for predicting the presence of optic nerve atrophy, making it a potential diagnostic tool for radiologists.

\section{REFERENCES}

1. Biousse V, Newman NJ. Diagnosis and clinical features of common optic neuropathies. Lancet Neurol 2016;15:1355-67 CrossRef Medline

2. Trip SA, Schlottmann PG, Jones SJ, et al. Optic nerve atrophy and retinal nerve fibre layer thinning following optic neuritis: evidence that axonal loss is a substrate of MRI-detected atrophy. Neuroimage 2006;31:286-93 CrossRef Medline

3. Mackay DD, Garza PS, Bruce BB, et al. The demise of direct ophthalmoscopy: a modern clinical challenge. Neurol Clin Pract 2015;5:150-57 CrossRef Medline

4. Behbehani R. Clinical approach to optic neuropathies. Clin Ophthalmol 2007;1:233-46 Medline

5. Šimundić AM. Measures of diagnostic accuracy: basic definitions. EJIFCC 2009;19:203-11 Medline

6. Hobom M, Storch MK, Weissert R, et al. Mechanisms and time course of neuronal degeneration in experimental autoimmune encephalomyelitis. Brain Pathol 2004;14:148-57 CrossRef Medline

7. Mead B, Tomarev S. Evaluating retinal ganglion cell loss and dysfunction. Exp Eye Res 2016;151:96-106 CrossRef Medline

8. Barrett K, Barman SM, Brooks S, et al. Ganong's Review of Medical Physiology. New York: McGraw Hill; 2010

9. Hee MR, Izatt JA, Swanson EA, et al. Optical coherence tomography of the human retina. Arch Ophthalmol 1995;113:325-32 CrossRef Medline

10. Maldonado RS, Mettu P, El-Dairi M, et al. The application of optical coherence tomography in neurologic diseases. Neurol Clin Pract 2015;5:460-69 CrossRef Medline

11. Wang XL, Yu T, Xia DZ, et al. Measurement of retinal nerve fiber layer thickness in optic atrophy eyes of patients with optic neuritis using optical coherence tomography. Graefes Arch Clin Exp Ophthalmol 2010;248:1013-18 CrossRef Medline

12. Karim S, Clark RA, Poukens V, et al. Demonstration of systematic variation in human intraorbital optic nerve size by quantitative magnetic resonance imaging and histology. Invest Ophthalmol Vis Sci 2004;45:1047-51 CrossRef Medline 